\title{
Accuracy of tracheal aspirate gram stain in predicting Staphylococcus aureus infection in ventilator-associated pneumonia
}

\author{
Renato Seligman ${ }^{1,2^{*}}$, Beatriz Graeff Santos Seligman ${ }^{1,2}$, Loriane Konkewicz ${ }^{1}$ and Rodrigo Pires dos Santos ${ }^{1}$
}

\begin{abstract}
Background: The Gram stain can be used to direct initial empiric antimicrobial therapy when complete culture is not available. This rapid test could prevent the initiation of inappropriate therapy and adverse outcomes. However, several studies have attempted to determine the value of the Gram stain in the diagnosis and therapy of bacterial infection in different populations of patients with ventilator-associated pneumonia (VAP) with conflicting results. The objective of this study is to evaluate the accuracy of the Gram stain in predicting the existence of Staphylococcus aureus infections from cultures of patients suspected of having VAP.

Methods: This prospective single-center open cohort study enrolled 399 patients from December 2005 to December 2010. Patients suspected of having VAP by ATS IDSA criteria were included. Respiratory secretion samples were collected by tracheal aspirate (TA) for standard bacterioscopic analysis by Gram stain and culture.

Results: Respiratory secretion samples collected by tracheal aspirates of 392 patients were analyzed by Gram stain and culture. When Gram-positive cocci were arranged in clusters, the sensitivity was $68.4 \%$, specificity $97.8 \%$, positive predictive value $88.1 \%$ and negative predictive value $92.8 \%$ for predicting the presence of Staphylococcus aureus in culture $(p<0.001)$.

Conclusions: A tracheal aspirate Gram stain can be used to rule out the presence of Staphylococcus aureus in patients with a clinical diagnosis of VAP with a 92.8\% Negative Predictive Value. Therefore, 7.2\% of patients with Staphylococcus aureus would not be protected by an empiric treatment that limits antimicrobial coverage to Staphylococcus aureus only when Gram positive cocci in clusters are identified.
\end{abstract}

Keywords: Staphylococcus aureus, Gram stain, Tracheal aspirate, Ventilator-associated pneumonia

\section{Background}

Ventilator Associated Pneumonia (VAP) has high mortality rates $[1,2]$, and thus, early empirical prescription of broad spectrum antibiotics is mandatory for these patients $[2,3]$. The trade-off for this practice is progressive induction of bacterial resistance in intensive care units (ICU). Aerobic Gram negative bacilli are the most frequent etiological agents in VAP [4]. Initial treatment decisions sought to identify clinical clues to suggest an etiology for VAP, such as invasive procedures, length of

\footnotetext{
* Correspondence: reseligman@hcpa.ufrgs.br

${ }^{1}$ Hospital de Clinicas de Porto Alegre, Rua Ramiro Barcelos 2350, 90035-003, Porto Alegre, Brazil

Universidade Federal do Rio Grande do Sul, Rua Ramiro Barcelos 2400 - 40 Andar, 90035-003, Porto Alegre, Brazil
}

stay, prior antimicrobial use, immunosuppressive states and ecological flora data [4]. The decision to consider MRSA and vancomycin prescription during initial treatment takes into account the presence of diabetes mellitus, head trauma [5], comorbid conditions and local microbiological patterns of the ICU. In this context, the tracheal aspirate Gram stain can be used to guide initial empiric antimicrobial therapy. This rapid test could prevent the initiation of inappropriate therapy when culture results are not available. However, several studies attempting to determine its precision in VAP management have shown conflicting results [6-11].

In our hospital, to restrict antibiotic overuse and prevent the induction of multidrug-resistant organisms and the associated costs, a protocol created by the local Hospital 
Infection Control Committee precludes the use vancomycin for the initial empiric treatment of VAP in the absence of gram-positive cocci by tracheal aspirate.

The objective of this study is to evaluate the accuracy of Gram staining bacterioscopic analysis in tracheal aspirate samples to predict Staphylococcus aureus presence or absence in cultures of suspected VAP patients.

\section{Methods}

This prospective single-center open cohort study enrolled patients admitted to a university teaching hospital ICU from December 2005 to December 2010. Three hundred ninety-nine patients suspected of having VAP by ATS IDSA criteria were included $[4,12]$. Respiratory secretion samples were collected by tracheal aspirate for standard bacterioscopic analysis by Gram stain and culture. Patients who did not achieve the criteria for VAP by the Clinical Pulmonary Infection Score (CPIS) $[2,13]$ were excluded. All patient data were prospectively collected by researchers of the Infection Control Committee and registered in a database. All samples were Gram stained by the microbiology technicians and read by hospital microbiologists. Samples with more than 10 epithelial cells per field under 100x magnification were discarded and new material was collected for analysis. After evaluating bacterial smears by Gram stain, cultures were plated on chocolate agar and, if necessary, on blood agar, azide blood agar and McConkey agar. Analysis of the colonies was performed in order to quantify, identify the pathogen and its antimicrobial susceptibility profile. A culture was considered to be positive when culture density reached more than $10^{5} \mathrm{CFU} / \mathrm{ml}$.

Statistical analysis: Data were analyzed using SPSS $^{\circ}$ 19 software (IBM, 2010). Relationships between the culture result and the Gram stain were tested with the chi-square test. Statistical significance was accepted at $P<0.05$.

The present study was approved by the Hospital de Clínicas de Porto Alegre Research Ethics Committee. The committee waived requirements for informed consent because it was obtained in a previous study with the same cohort.

\section{Results}

The study design is shown in Figure 1.

Samples of respiratory secretions collected by tracheal aspirates of 392 patients were analyzed by Gram stain and culture.

Gram positive cocci, including in clusters, chains and diplococci, were observed upon Gram staining of 148 (37.7\%) samples.

Gram positive cocci in clusters were identified in 59 (15.1\%) samples, and $52(88.1 \%)$ of these contained Staphylococcus aureus isolated from the cultures.
Cross tabulation of the Gram stain and Staphylococcus aureus results in tracheal aspirate cultures is shown in Table 1.

The accuracy of the Gram stain in predicting Staphylococcus aureus in tracheal aspirate samples is shown in Table 2.

When Gram-positive cocci were arranged in clusters, the sensitivity was $68.4 \%$, specificity $97.8 \%$, positive predictive value $88.1 \%$ and negative predictive value $92.8 \%$ for predicting the presence of Staphylococcus aureus in culture $(p<0.001)$.

Twenty eight (7.1\%) samples contained Gram positive cocci in chains, and five of these contained Staphylococcus aureus isolated in culture. There was no statistical correlation between the presence of gram positive cocci in chains and the growth of Staphylococcus aureus in culture.

From all Staphylococcus aureus samples identified, $71.1 \%$ were methicillin-resistant.

\section{Discussion}

In our study, we found a Positive Predictive Value of $82.1 \%$ and a Negative Predictive Value of $92,8 \%$ for the Gram stain in identification of Staphylococcus aureus in tracheal aspirate samples.

The high Negative Predictive Value observed in this study is in accordance with the results of Blot et al. [6]. When considering microbiologically confirmed VAP, these authors found that the sensitivity and specificity of the Gram stain examination were $89 \%$ and $56 \%$, respectively, for the tracheal aspirate. The negative and positive predictive values of Gram stain examination for tracheal aspirate were $90 \%$ and $53 \%$, respectively. Their results strongly suggest that when the Gram stain examination of tracheal aspirate is negative, the diagnosis of VAP is very unlikely. Similarly, Fagon et al. [14] demonstrated that Gram stain examination of the tracheal aspirate showed a good sensitivity $(88,9 \%)$ but lacked specificity (59.6\% false positives) in the diagnosis of VAP.

Other authors describe different results. Namias et al. [9] showed poor overall correlation between Gram positive cocci with the aspirate culture results in a study in which the Gram stain was used to guide empiric antibiotic treatment of pneumonia in surgical ICU patients. Tetenta and Metersky [11] found that the Gram stain had a sensitivity of $68 \%$, a specificity of $72 \%$, a negative predictive value of $80 \%$ and a positive predictive value of $59 \%$ for Staphylococcus aureus. However, the authors did not attempt to correlate the Gram stain findings with the presence of VAP. Therefore, some of these patients may have had low level colonization but not Staphylococcus aureus pneumonia.

Considering these results, providers are challenged to determine the level of acceptable risk associated with 


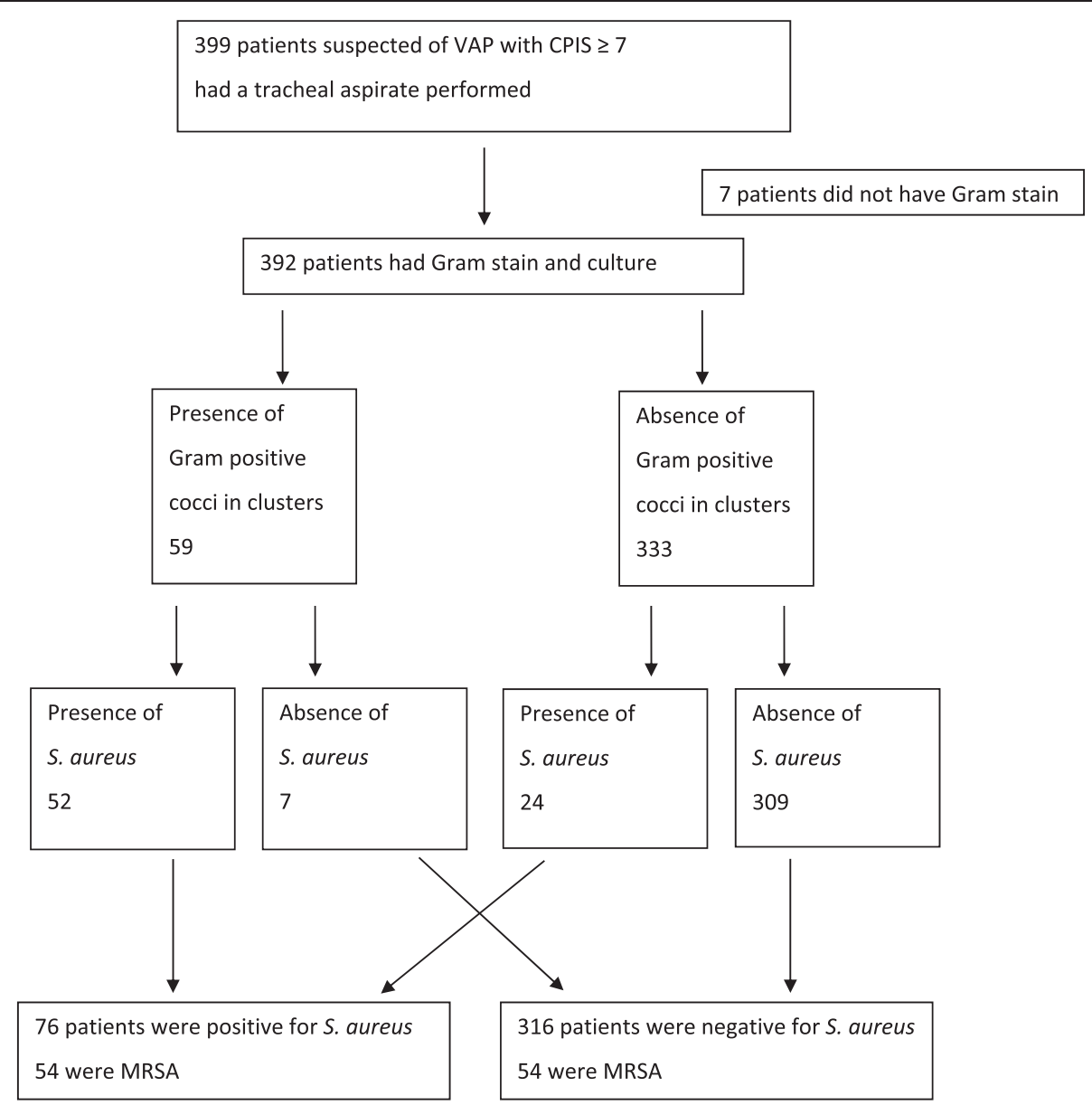

Figure 1 The study design. VAP, ventilator-associated pneumonia; CPIS, clinical pulmonary infection score; MRSA, methicillin- resistant Staphylococcus aureus; S. aureus, Staphylococcus aureus.

misdiagnosing a condition that has a mortality ranging between 25 to $76 \%[3,4]$. It is well established that early and appropriate empirical treatment reduces mortality [15-18]. The results of culture tests usually take 72 hours to become available, which could represent an unnecessary delay in determining the appropriate treatment.

Some evidence demonstrates that waiting for culture results may have important consequences for the patient. Iregui et al. [16] demonstrated that patients who met VAP diagnostic criteria and waited for culture results to initiate treatment experienced significant delays in receiving antibiotics. The mean time interval until the

Table 1 Cross tabulation of the Gram stain and Staphylococcus aureus in the tracheal aspirate

\begin{tabular}{lllll}
\hline & & $\begin{array}{l}\text { Staphylococcus } \\
\text { Yes }\end{array}$ & $\begin{array}{l}\text { Aureus } \\
\text { No }\end{array}$ & Total \\
\hline Gram Positive & Yes & 52 & 7 & 59 \\
in clusters & No & 24 & 309 & 333 \\
& Total & 76 & 316 & 392 \\
\hline
\end{tabular}

administration of antibiotic treatment was $28.6 \mathrm{~h}$ among patients who waited for culture results, compared to $12.5 \mathrm{~h}$ for all other patients $(\mathrm{p}<0.001)$. Delayed administration of antibiotics was an independent risk factor for mortality (odds ratio, 7.68; 95\%CI, $4.50-13.09$; $\mathrm{p}<0.001$ ).

Luna et al. [18] demonstrated that when adequate antibiotic therapy is initiated very early (before performing

Table 2 Accuracy of Gram stain in predicting Staphylococcus aureus in the tracheal aspirate

\begin{tabular}{ll}
\hline Sensitivity & 68.4 \\
Specificity & 97.8 \\
Positive Predictive Value & 88.1 \\
Negative Predictive Value & 92.8 \\
Positive Likelihood Ratio & 31.1 \\
Negative Likelihood Ratio & 0.3 \\
Pre-Test Probability & 19.4 \\
Post-Test Probability - Positive & 88.2 \\
Post-Test Probability - Negative & 7.2 \\
\hline Pearson Chi Square $p=0.0001$. &
\end{tabular}


bronchoscopy), the mortality rate is reduced (38\%) when compared to inadequate or absent therapies (91\%) ( $\mathrm{p}<$ 0.001). When patient treatment regimens were improved to adequate antibiotic therapy, mortality was comparable to those who continued to receive inadequate therapy based on BAL results. Kollef and Ward [17] showed that changing or modifying initially inadequate antibiotic treatment did not improve patient outcomes. The hospital mortality rate of patients who have their antibiotic therapy changed or whose therapy started following culture was $61 \%$, while the mortality rate of patients who did not experience any changes in their antibiotic management was $33 \%$. These treatment modifications likely occurred too late in the course of the illness to have a beneficial effect.

Rello and coworkers [19] also found a significant increase in mortality as a result of inappropriate early antibiotic therapy (37 versus 15\%) despite guided changes in therapy after the results of cultures.

Based on these reports, it seems unquestionable that antibiotic treatment must be started early and that late escalation of treatment offers no clear advantage. Currently, Gram staining could be an useful tool if it provides accurate results. In this context, our findings demonstrate that to rule out the presence of Staphylococcus aureus, the Gram stain offered a Negative Predictive Value of $92.8 \%$. Therefore, $7.2 \%$ of patients with Staphylococcus aureus would not be covered by an empiric treatment that precludes the use of vancomycin when there is not identification of Gram-positive cocci in clusters in the tracheal aspirate. The microbial ecology of the unit and clinical conditions should also be considered in the therapeutic decision. In settings with high incidence of MRSA infections, avoidance of empirical vancomycin usage increases the risk of inappropriate treatment.

Our study has some limitations. It is a single center study, and we did not include patients suspected of VAP with negative cultures. Similar to other studies, many samples of the lower respiratory tract were obtained from patients who had received previous or current antibiotics, potentially influencing the culture results. However, to establish the Positive Predictive Value and Negative Predictive Value, it is necessary to understand the true prevalence of infection, which requires a patient population with microbiologically confirmed VAP. Another limitation is the accuracy of clinical diagnosis of VAP with CPIS $\geq 7$ confirmed by positive culture and the 2005 ATS IDSA diagnosis guidelines used in this study, which have been recently updated [20]. The prospective nature of our study is an advantage.

\section{Conclusions}

Gram staining of the tracheal aspirate can rule out the presence of Staphylococcus aureus in patients with a clinical diagnosis of VAP with a 92.8\% Negative Predictive Value. Therefore, $7.2 \%$ of patients with Staphylococcus aureus would not be protected by an empiric treatment that precludes the use vancomycin when Grampositive cocci in clusters are not identified in the tracheal aspirate.

\section{Abbreviations}

CFU: Colony-forming unit; CPIS: Clinical pulmonary infection score; ICU: Intensive care unit; MRSA: Methicillin-resistant Staphylococcus aureus; S. aureus: Staphylococcus aureus; TA: Tracheal aspirate; VAP: Ventilatorassociated pneumonia.

\section{Competing interests}

The authors declare that they have no competing interests.

\section{Authors' contributions}

RS contributed substantially towards the conception and design, manuscript drafting, analysis and interpretation of data, and critically revising the manuscript for intellectual content and final approval. BGSS was involved in study design, drafting and revising the work for intellectual content and final approval. LRK contributed substantially towards the conception and design of the work and the acquisition, analysis and interpretation of data. RPS contributed substantially towards the conception, design of the study and the acquisition, analysis, and interpretation of data. All authors read and approved the final manuscript.

\section{Acknowledgements}

The authors would like to thank the support from Fundo de Incentivo à Pesquisa (FIPE, Research Incentive Fund) of Hospital de Clínicas de Porto Alegre. There was no involvement of the funding resource in research design, collection, analysis or interpretation of data. The writing and publication of the manuscript were completed by the authors without any participation or influence from the funding source. This study was approved by the Research Ethics Committee of Hospital de Clínicas de Porto Alegre, Universidade Federal do Rio Grande do Sul.

Grupo de Pesquisa em Pneumonias - HCPA - CNPq, Hospital de Clinicas de Porto Alegre, Universidade Federal do Rio Grande do Sul, Porto Alegre/RS, Brazil.

Funded by Fundo de Incentivo a Pesquisa do Hospital de Clinicas de Porto Alegre, FIPEHCPA, Porto Alegre, Brazil.

Received: 8 August 2014 Accepted: 13 January 2015 Published: 23 January 2015

\section{References}

1. Muscedere JG, Martin CM, Heyland DK. The impact of ventilator-associated pneumonia on the Canadian health care system. J Crit Care. 2008;23(1):5-10.

2. Raghavendran K, Wang J, Belber C, Misra SR, Brunton K, Berbary E, et al. Predictive value of sputum gram stain for the determination of appropriate antibiotic therapy in ventilator-associated pneumonia. J Trauma. 2007;62(6):1377-82. discussion 1382-1373.

3. Chastre J, Fagon JY. Ventilator-associated pneumonia. Am J Respir Crit Care Med. 2002;165(7):867-903.

4. American Thoracic Society (ATS), Infectious Diseases Society of America (IDSA). Guidelines for the management of adults with hospital-acquired, ventilator-associated, and healthcare-associated pneumonia. Am J Respir Crit Care Med. 2005;171(4):388-416.

5. Rello J, Torres A, Ricart M, Valles J, Gonzalez J, Artigas A, et al. Ventilatorassociated pneumonia by Staphylococcus aureus. Comparison of methicillin-resistant and methicillin-sensitive episodes. Am J Respir Crit Care Med. 1994;150(6 Pt 1):1545-9.

6. Blot F, Raynard B, Chachaty E, Tancrede C, Antoun S, Nitenberg G. Value of gram stain examination of lower respiratory tract secretions for early diagnosis of nosocomial pneumonia. Am J Respir Crit Care Med. 2000;162(5):1731-7.

7. Davis KA, Eckert MJ, Reed 2nd RL, Esposito TJ, Santaniello JM, Poulakidas S, et al. Ventilator-associated pneumonia in injured patients: do you trust your Gram's stain? J Trauma. 2005;58(3):462-6. discussion 466-467. 
8. Goldberg AE, Malhotra AK, Riaz OJ, Aboutanos MB, Duane TM, Borchers CT, et al. Predictive value of broncho-alveolar lavage fluid Gram's stain in the diagnosis of ventilator-associated pneumonia: a prospective study. J Trauma. 2008;65(4):871-6. discussion 876-878.

9. Namias N, Harvill S, Ball S, McKenney MG, Sleeman D, Ladha A, et al. A reappraisal of the role of Gram's stains of tracheal aspirates in guiding antibiotic selection in the surgical intensive care unit. J Trauma. 1998:44(1):102-6.

10. O'Horo JC, Thompson D, Safdar N. Is the gram stain useful in the microbiologic diagnosis of VAP? A meta-analysis. Clin Infect Dis. 2012:55(4):551-61.

11. Tetenta S, Metersky ML. Tracheal aspirate Gram stain has limited sensitivity and specificity for detecting Staphylococcus aureus. Respirology. 2011;16(1):86-9.

12. Craven DE, Kunches LM, Kilinsky V, Lichtenberg DA, Make BJ, McCabe WR. Risk factors for pneumonia and fatality in patients receiving continuous mechanical ventilation. Am Rev Respir Dis. 1986;133(5):792-6.

13. Tablan OC, Anderson L, Besser R, Bridges C, Hajjeh R. Guidelines for preventing health-care--associated pneumonia, 2003: recommendations of CDC and the Healthcare Infection Control Practices Advisory Committee. MMWR Recomm Rep. 2004;53(RR-3):1-36.

14. Fagon JY, Chastre J, Wolff M, Gervais C, Parer-Aubas S, Stephan F, et al. Invasive and noninvasive strategies for management of suspected ventilator-associated pneumonia. A randomized trial. Ann Intern Med. 2000;132(8):621-30.

15. Alvarez-Lerma F. Modification of empiric antibiotic treatment in patients with pneumonia acquired in the intensive care unit. ICU-Acquired Pneumonia Study Group. Intensive Care Med. 1996;22(5):387-94.

16. Iregui M, Ward S, Sherman G, Fraser VJ, Kollef MH. Clinical importance of delays in the initiation of appropriate antibiotic treatment for ventilatorassociated pneumonia. Chest. 2002;122(1):262-8.

17. Kollef MH, Ward S. The influence of mini-BAL cultures on patient outcomes: implications for the antibiotic management of ventilator-associated pneumonia. Chest. 1998;113(2):412-20.

18. Luna CM, Vujacich P, Niederman MS, Vay C, Gherardi C, Matera J, et al. Impact of BAL data on the therapy and outcome of ventilator-associated pneumonia. Chest. 1997;111(3):676-85.

19. Rello J, Gallego M, Mariscal D, Sonora R, Valles J. The value of routine microbial investigation in ventilator-associated pneumonia. Am J Respir Crit Care Med. 1997;156(1):196-200.

20. Magill SS, Klompas M, Balk R, Burns SM, Deutschman CS, Diekema D, et al. Developing a new, national approach to surveillance for ventilatorassociated events. Am J Crit Care. 2013;22(6):469-73.

doi:10.1186/1471-2253-15-19

Cite this article as: Seligman et al:: Accuracy of tracheal aspirate gram stain in predicting Staphylococcus aureus infection in ventilatorassociated pneumonia. BMC Anesthesiology 2015 15:19.

\section{Submit your next manuscript to BioMed Central and take full advantage of:}

- Convenient online submission

- Thorough peer review

- No space constraints or color figure charges

- Immediate publication on acceptance

- Inclusion in PubMed, CAS, Scopus and Google Scholar

- Research which is freely available for redistribution 